\section{On categories of behavior and control groups in multiple-response procedures}

\author{
PHILIP J. DUNHAM \\ Dalhousie University, \\ Halifax, Nova Scotia B3H 4JI, Canada
}

I think that it is accurate to state that three basic criticisms can be extracted from Herring's (1979) comments on my recent punishment experiments (1978).

(1) She takes exception to our punishment rule which implies that all categories of behavior can be suppressed by punishment without regard for their functional relevance. Her argument is that we have punished only one category, traditionally thought of as "consummatory behavior," and that this does not constitute an adequate data base upon which to make such a sweeping generalization. There are several ways one might reply to this comment. I could, for example, point out that I did not make the sweeping generalization of which I have been accused; instead, I merely indicated that all of the responses we have punished in our research thus far have declined in probability and we have not yet had to resort to discussions of functional relevance to explain the data. Indeed, none of our punishment research has been specifically designed to test the notion that speciesspecific defensive responses are immune to punishment contingencies. However, such a reply (while somewhat accurate) does not make for very interesting polemics, and it probably does betray the intuitive confidence I have in the generality of the punishment rule.

I might also reply that the weight of the evidence in the literature does, in my opinion, indicate that responses which are punished do tend to decline without much respect for categories such as defensive, consummatory, instrumental, instinctive, etc.

I am, however, hesitant to make either of the above replies because they both beg a more fundamental question which must be dealt with before either Herring's comments or my intuitions can be experimentally tested. Specifically, the minimum requirement which must be met if one is to test the notion that certain categories of responding will be immune to punishment is that these special categories must be operationally defined. Consider the proposition that species-specific defensive responses are immune to punishment. Now consider pain-elicited attack behavior as a response. If I demonstrate, as Azrin (1970) has, that response-contingent shocks will suppress pain-elicited attack responses have I tested the proposition? I can't answer because none of the terms in the proposition have been operationally defined. My zoologist friends argue at length over how one might operationally define the term "species"; proponents of movement notation systems tell me that I shouldn't be using such linguistically convenient labels as "attack" to describe the stream of motor output in my multiple-response environments; and I guess I am suggesting to Herring that I also have trouble determining whether pain-elicited attack is a "consummatory" or a "defensive" behavior. Until these definitional problems are solved, it doesn't seem to make too much sense to argue over whether any specific data question the proposition that certain categories of behavior are immune to punishment. I have made this particular argument in more detail in an earlier manuscript (Dunham, 1977).

(2) Herring also takes exception to our generalization which suggests that only the most probable of the unpunished responses in the repertoire will increase during punishment. Again, her argument is that we have typically observed only one category of unpunished behavior-species-specific defensive reactions such as running - and that this does not constitute an adequate data base for the proposed generalization. I should point out that I don't have much faith in this generalization surviving future data either, but at present the criticism is not quite accurate. We have observed gerbils increase their paper-shredding behavior when eating was punished and rats increase polydipsic drinking when leverpressing was punished (Dunham, 1971). Again, however, I hesitate to make this reply because it begs the same fundamental question that was discussed earlier. Until these intuitive categories of behavior are operationally defined, the suggestion that a particular response is exempt from the generalization cannot be decisively tested. Consequently, our punishment research has not been designed to answer this question, although I do admit to occasionally begging this question in discussing the results!

(3) Finally, Herring suggests that we have not run an appropriate control group (i.e., noncontingent shock) to separate the "associative" and "nonassociative" effects of the response-shock contingency. Can the decline in punished responding and the increase in the most probable unpunished alternative be viewed as simply "nonassociative" effects of aversive stimulation? Perhaps the most direct reply to this criticism is to plead guilty. As Herring states, we have not yet concerned ourselves with the question of "associative" and "nonassociative" processes in our analysis of multiple response adjustments to aversive stimulation. There are, however, several reasons why we have not yet introduced this traditional control procedure (i.e., noncontingent shock) into our research using 
the multiple-response paradigm. Perhaps the most substantive reason is that it seems a dangerous oversimplification to suggest that a group receiving noncontingent shock would assess the "nonassociative" properties of this aversive event. If you start from the assumption that behavior fills time (as we do in the multiple-response analysis), you are immediately confronted with the difficult question of how to deliver the "noncontingent" shocks. Herring does not make specific suggestions on this point, but given limited space, one example should illustrate the complexity of this problem (the reader can pursue other variations on the theme).

The traditional solution to the problem in the context of operant leverpressing behavior would be to use a "yoked-control procedure." Yoking is generally considered to have the fewest confounding problems in most traditional operant preparations. Consider, however, its implications in a multiple-response baseline procedure consisting of baseline responses $\mathrm{A}$, $B, C, D$, and a response $E$ which is elicited each time the control animal receives a "yoked" shock. Under these conditions, the elicited response, E, enjoys the unique property in the repertoire of predicting a longer safe interval than any other response. Indeed, as the punished response declines in the experimental animal, the yoked animal receives fewer shocks with longer intervals between them; hence the elicited behavior is essentially participating in an implicit avoidance contingency very much like the traditional Sidman avoidance procedure. Consequently, we would find ourselves using an avoidance contingency procedure to assess the "nonassociative" effects of shock-a procedure conceptually analagous to using backward pairing as a control for "nonassociative" effects in a Pavlovian excitatory conditioning procedure. We have, in fact, recently demonstrated that shock-elicited behaviors are sensitive to this implicit arrangement (cf. Dunham \& Carr, 1976).

In thinking about the problem of controls for "nonassociative" effects in the multiple-response paradigm, I have reached the following conclusions at this point in time: (1) I agree with Herring that the effects of noncontingent shock should be studied in the multiple-response paradigm, not, however, as a simple control procedure for "nonassociative" effects, but as a set of operations sui generis. I think the yoked control example illustrates why this would be the most analytic approach to the problem of "associative" vs. "nonassociative" effects, given that one wishes to conceptualize the behavioral changes in this way. (2) I am very much taken with Rachlin and Burkhard's (1978) recent admonishment that: "What is usually thought of as instrumental behavior can be explained without the concepts of reinforcement and punishment as they were originally intended, that is, a strengthening of a connection ... or a response ... by temporal contiguity" (p. 44). Indeed, history might eventually reveal that a long search for the "appropriate" control for "nonassociative" effects in this context contributed substantially to the demise of those theoretical models which forced the question in the first place!

\section{REFERENCES}

Azrin, N. Punishment of elicited aggression. Journal of the Experimental Analysis of Behavior, 1970, 14, 7-10.

Dunham, P. J. Punishment: Method and theory. Psychological Review, 1971, 78, 58-70.

Dunham, P. J. Changes in unpunished responding during response-contingent punishment. Animal Learning \& Behavior, $1978,6,174-180$.

Dunham, P. J. The nature of reinforcing stimuli. In V. Honig \& J. Staddon (Eds.), Handbook of operant behavior. Englewood Cliffs, N.J: Prentice-Hall, 1977.

Dunham, P. J., \& CARR, A. Pain-elicited aggression in the squirrel monkey: An implicit avoidance contingency. Animal Learning \& Behavior, 1976, 4, 89-95.

Herring, B. Comments on paper by Dunham. Animal Learning \& Behavior, 1979, 7, 271-272.

RaChlin, H., \& BurKhaRd, B. The temporal triangle: Response substitution in instrumental conditioning. Psychological Review, 1978, 85, 22-47.

(Received for publication November 8, 1978; accepted November 13, 1978.) 